\title{
Pregnant Women Urged to Get COVID-19 Vaccine
}

Michelle Winokur, DrPH, and the AfPA Governmental Affairs Team, Alliance for Patient Access (AfPA)

The Alliance for Patient Access (allianceforpatientaccess.org), founded in 2006, is a national network of physicians dedicated to ensuring patient access to approved therapies and appropriate clinical care. AfPA accomplishes this mission by recruiting, training and mobilizing policy-minded physicians to be effective advocates for patient access. AfPA is organized as a non-profit 501(c)(4) corporation and headed by an independent board of directors. Its physician leadership is supported by policy advocacy management and public affairs consultants. In 2012, AfPA established the Institute for Patient Access (IfPA), a related 501(c) (3) non-profit corporation. In keeping with its mission to promote a better understanding of the benefits of the physician-patient relationship in the provision of quality healthcare, IfPA sponsors policy research and educational programming.

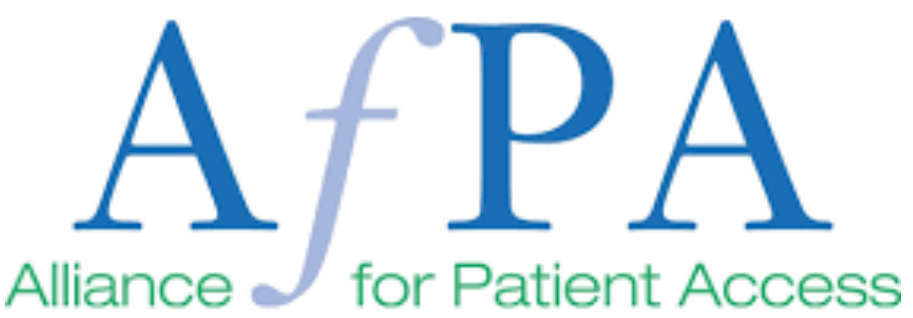

"A federal health advisory from the Centers for Disease Control and Prevention follows the release of new data showing a $70 \%$ increased risk of death from COVID-19 during pregnancy. Unvaccinated pregnant women also have a higher risk of early delivery or stillbirth."

Public health officials are urging pregnant and breastfeeding moms to get the COVID-19 vaccine - and soon.

A federal health advisory from the Centers for Disease Control and Prevention follows the release of new data showing a $70 \%$ increased risk of death from COVID-19 during pregnancy. Unvaccinated pregnant women also have a higher risk of early delivery or stillbirth.

Pregnancy and Vaccine Safety
As part of their campaign to encourage pregnant women to get inoculated, Federal health officials are highlighting the safety and efficacy of the vaccine. As the health advisory notes, the vaccine does not increase the risk of miscarriage or birth defects or affect fertility.

The COVID-19 vaccine is recommended for pregnant women, recently pregnant, breastfeeding, or trying to get pregnant. Professional medical organizations have endorsed these recommendations, including the American College of Obstetricians and Gynecologists and the Society for Maternal-Fetal Medicine.

\section{COVID-19 Among Pregnant Women}

Despite the benefits to mothers and their unborn babies, only $31 \%$ of pregnant women are vaccinated against COVID-19. Being unvaccinated leaves expectant moms vulnerable to contracting $\mathrm{CO}$ VID-19, while pregnancy makes them more likely to experience severe symptoms and require intensive-level care.

\section{"Thus far, approximately $97 \%$ of pregnant women hospitalized with COVID-19 were unvaccinated. Moreover, in August, 22 pregnant women died of COVID-19, making it the single highest month for COVID-related pregnancy deaths since} the pandemic began."

Thus far, approximately $97 \%$ of pregnant women hospitalized with COVID-19 were unvaccinated. Moreover, in August, 22 pregnant women died of COVID-19, making it the single highest month for COVID-related pregnancy deaths since the pandemic began.

In contrast, getting vaccinated protects both expectant moms and her baby. Getting vaccinated is the single most effective way to prevent serious illnesses, death, and adverse pregnancy outcomes from the coronavirus.

\section{A Precaution Not to be Overlooked}

Women who are planning to get pregnant take many steps to prepare. Likewise, expectant moms pay extra attention to their health and safety for the sake of their babies.

Getting vaccinated against COVID-19 is one precaution they should not overlook. The sooner, the better, say the experts.

\section{References:}

https://emergency.cdc.gov/han/2021/han00453.asp

Disclosures: Michelle Winokur, DrPH, is the Policy Communications Director for the Alliance for Patient Access. 


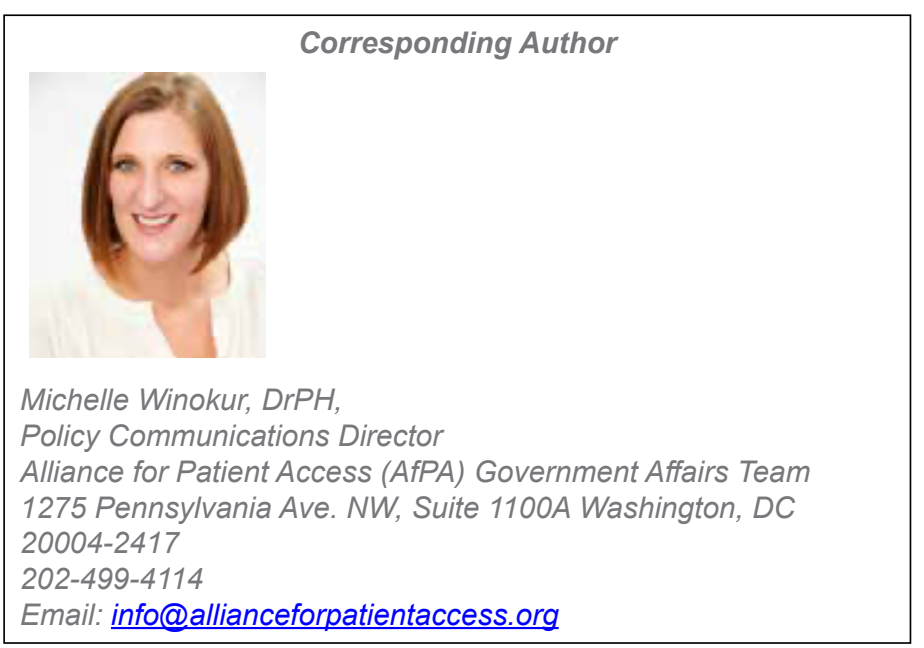

TAKE THE NECESSARY STEPS TO

\section{ELIMINATE INEQUITIES}
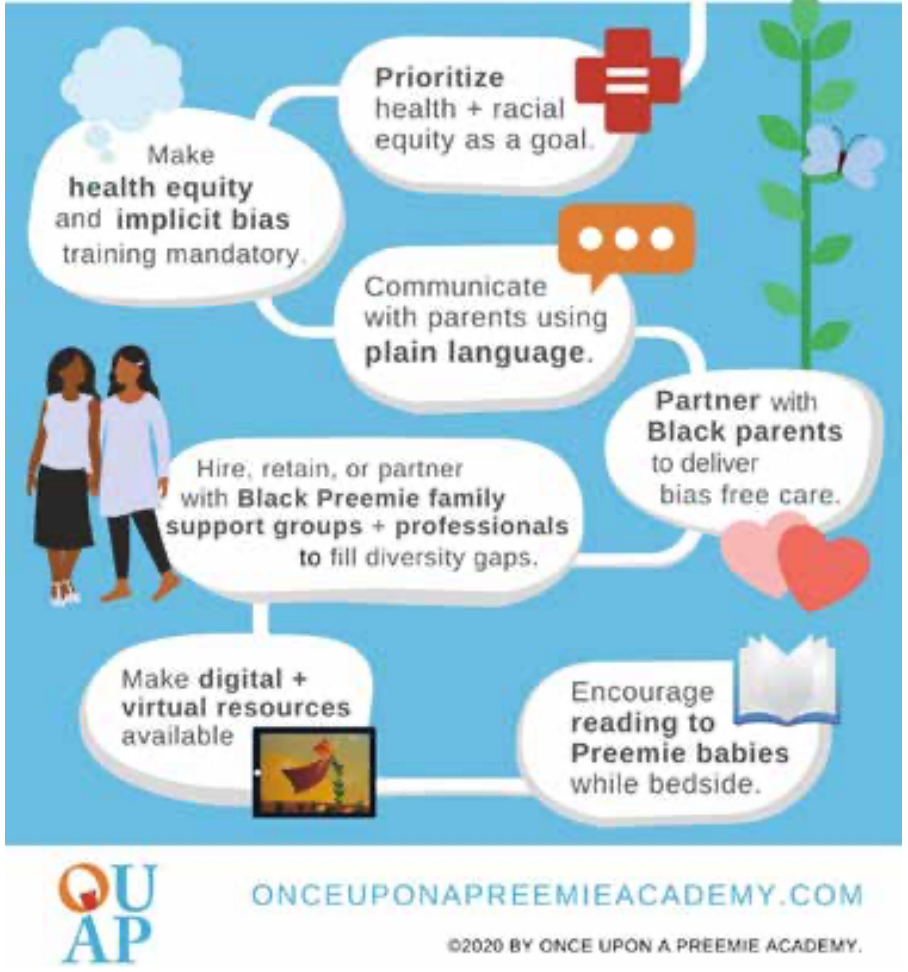

\section{Readers can also follow \\ NEONATOLOGY TOL \\ via our Twitter Feed \\ @NEOTODAY}

\section{Still a Preemie?}

Some preemies are born months early, at extremely low

birthweights. They fight for each breath and face nearly insurmountable health obstacles.

But that's not every preemie's story.

Born between 34 and 36 weeks gestation?

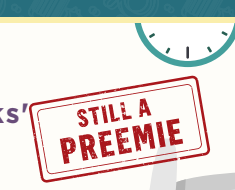

Just like preemies born much earlier, these "late preterm" infants can face:

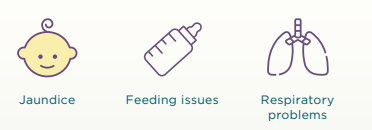

And their parents, like all parents of preemies, are at risk for postpartum depression and PTSD.

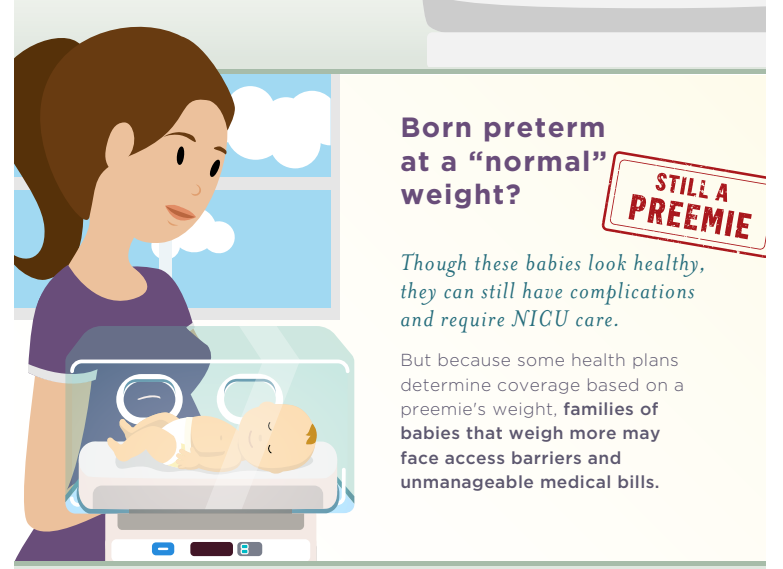

Born preterm but not admitted to the NICU?

Even if preterm babies

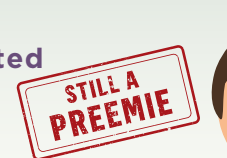
don't require NICU care,

they can still face health challenges.

Those challenges can extend through childhood, adolescence and even into adulthood.
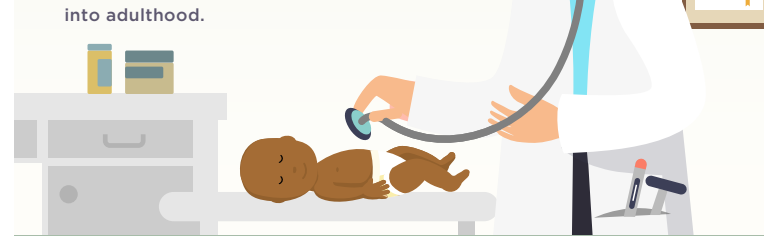

Some Preemies

Will spend weeks

调 in the hospital

-1. Will have lifelong |था from birth
解 Are disadvantaged
All Preemies

F Face health

$\oplus$ Deserve appropriate health coverag

Uo $\begin{aligned} & \text { Need access to } \\ & \text { proper health car }\end{aligned}$

\section{$N C f I H$ Nartional Coalition \\ www.infanthealth.org}

